\section{Research Square}

Preprints are preliminary reports that have not undergone peer review. They should not be considered conclusive, used to inform clinical practice, or referenced by the media as validated information.

\title{
Bacterial Tonsillitis and Antimicrobial Resistance Profiles Among Children Within Five Years of Age At Hargeisa Group of Hospital, Somaliland: A Cross-Sectional Study
}

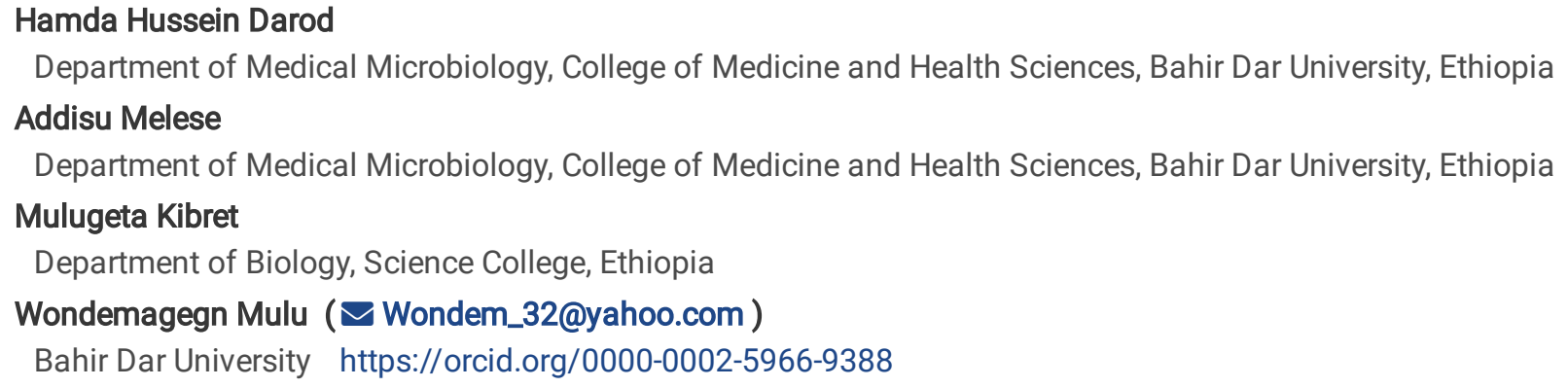

\section{Research Article}

Keywords: Children, tonsillitis, bacteria, antimicrobial resistance, Hargeisa group Hospital

Posted Date: June 12th, 2021

DOI: https://doi.org/10.21203/rs.3.rs-586545/v1

License: (a) (i) This work is licensed under a Creative Commons Attribution 4.0 International License. Read Full License 


\section{Abstract \\ Background}

Tonsillitis is the third most frequently diagnosed infection in the pediatrics age group around the world. It causes significant morbidity and loss of school attendance in children. The emergence of drug resistance in bacterial tonsillitis is getting higher every year. However, data on the drug resistance profiles of bacterial causes of tonsillitis among children within five years of age is not available in Somaliland.Therefore; this study determined the bacterial causes of tonsillitis and their antimicrobial resistance profiles among children within five years of age at Hargeisa Group of Hospital, Somaliland.

\section{Methods}

A cross-sectional study was conducted from March to July 2020. A total of 374 children within five years of age were included using convenient sampling method. Throat swabs were collected from children, processed and bacterial species were identified using standard bacteriological procedures. Antimicrobial susceptibility was done using disc diffusion method. Data on demographic variables and clinical profiles were collected using structured questionnaires. Logistic regression analysis was computed to identify factors associated with bacterial tonsillitis. P-values $<0.05$ were taken as statistically significant.

\section{Results}

The median age of children included in the study was 4 years. Overall, $120(32.1 \%)(95 \% \mathrm{Cl} 27.4-36.8 \%)$ of children had culture confirmed bacterial tonsillitis. Of them,23(19.2\%) had mixed infections. The most frequent bacterial isolates were Streptococcus pyogenes 78(55\%), Staphylococcus aureus 42 (29\%) and Streptococcus pneumoniae 10(7\%). Isolates revealed $83.3-100 \%$ rate of resistance to ampicillin. S. aureus was resistant to clarithromycin (38\%) while $60 \%$ of $S$. pneumoniae isolates were resistant to gentamicin. The overall multidrug resistance (MDR) was $50.4 \%$ and $52.6 \%$ of S.pyogenes and $60 \%$ of S.pneumoniae were MDR. History of tonsillitis $(\mathrm{AOR}=0.12 ; 95 \% \mathrm{Cl}=0.06-0.21)$, difficulty of swallowing (AOR $=6.99 ; 95 \% \mathrm{Cl}=3.56-13.73$ ), weight loss (AOR = $0.33 ; 95 \% \mathrm{Cl}=0.186-0.597)$ and attending school $(A O R=2.98 ; 95 \% \mathrm{Cl}=1.64-5.42)$ were found to be associated with tonsillitis among children within five years of age.

\section{Conclusions}

Bacterial tonsillitis with high degree of ampicillin resistance, mixed infections and MDR isolates are major concerns in children within five years of age at Hargeisa, Somaliland. Therefore, treatment of cases should be guided by regular culture and antimicrobial susceptibility testing to prevent disease consequences and critical drug resistance.

\section{Background}

Tonsillitis is the inflammation of tonsils. It is contagious, and can spread through close contact with infected persons, sharing food, drinks and utensils. Tonsillitis impacts the health of children, their quality of life and causes significant morbidity and loss of time for schooling [1]. Poor living conditions, exposure to environmental pollutants and indoor air pollution are frequently reported factors associated with tonsillitis among children within five years of age [2].

Gram positive and Gram negative bacteria are frequently associated with symptomatic childhood tonsillitis. Of them, Streptococcus pyogenes (S. pyogenes), methicillin resistant Staphylococcus aureus (MRSA), Streptococcus pneumoniae(S. pneumoniae), Haemophilus influenzae (H. influenzae), Moraxella catarrhalis (M. catarrhalis), Pseudomonas aeruginosa (P. aeruginosa) and Klebsiella pneumoniae (K. pneumoniae) are the common etiologies of tonsillitis [3].

The emergence of drug resistant bacteria in tonsillitis is getting higher every year. Methicillin resistant $S$. aureus (MRSA) is one of the most frequent bacterial causes of tonsillitis in children. Irrational use of antibiotics by patients, production of beta-lactamase enzymes and formation of biofilms are the main reasons for emergence of drug resistance [4]. The spread of drug resistant bacteria has led to treatment failure and recurrence of tonsillitis among children with poor sanitation and hygiene in underdeveloped countries.

Page 2/22 
The situation is critical in Somaliland, where antimicrobials are vastly and frequently used irrationally. This might increase the emergence of resistance to the commonly used antibiotics for the treatment of tonsillitis [5].

In Hargeisa Group of Hospitals, like other similar health settings in Somaliland, routine culture and antibiotic susceptibility testing are not usually performed as essential part of patient care and treatments are mostly based on empirical therapy. However, there are no studies conducted and published on the culture confirmed burden of bacterial tonsillitis and antimicrobial resistance profiles as well as the extent of MRSA in children within five years of age in Somaliland. Moreover, asymptomatic children can be the sources of dissemination of bacteria causing tonsillitis to non-infected children at home or at school settings that can lead to wide range of tonsillar infections. Therefore, we present the first report of the prevalence of bacterial causes of tonsillitis and antimicrobial resistance profiles of the isolates in children within five years of age at Hargeisa Group of Hospital, Somaliland.

\section{Methods}

\section{Study design, period and setting}

A hospital based cross-sectional study was conducted between March and July 2020 in Hargeisa Group of Hospital (HGH), Somaliland. Hargeisa Group of Hospital is located in Maroodi Jeex Region, the capital city of Somaliland known as Hargeisa. According to the 2019 census report from Central Statistics Department of Somaliland, Hargeisa has a total population of 1.2 million. Hargeisa Group of Hospital is the largest referral public hospital with more than 200 healthcare professionals. It is one of the health hubs in Somaliland. The pediatric department of HGH has outpatient and inpatient departments with 15 Pediatricians and two Nurses. Daily 50 outpatients and 1 to 4 hospitalized children attend the Pediatric Clinic for different medical conditions. All children within five years of age with tonsillitis at Ear, Nose and Throat (ENT) of HGH were the study population.

\section{Inclusion and Exclusion Criteria}

Children within five years of age presumptive for tonsillitis with sore throat, red swollen tonsils, pain when swallowing, fever, cough, tiredness, malaise, and white pus-filled spots on the tonsils, swollen lymph nodes, pain in the ears or neck, and weight loss were included in the study. On the other hand, children who were on antibiotic treatment within the previous two weeks of sample collection, and had tonsillectomy were excluded from the study

\section{Sample size and Sampling}

The sample size was calculated using single population formula $n=(Z a / 2)^{2} P(1-P) / d^{2}$ where, $n=$ sample size, $Z=$ level of confidence according to the standard normal distribution, $P=$ sample proportion and $d=$ tolerated margin of error. Therefore; by taking $Z(\alpha / 2)=$ 1.96 for a level of confidence of $95 \%, P=0.5$ which is the maximum proportion of bacterial tonsillitis and $5 \%$ margin of error, the sample size was calculated as $\mathrm{n}=(1.96)^{2} \times 0.5(1-0.5) /(0.05)^{2}=384$. However, due to the lack of sufficient throat swabs and incomplete questionnaire based data, only 374 children within five years of age with tonsillitis were included in the study. Study participants were included conveniently. All children within five years of age with tonsillitis attending at ENT department of HGH and who fulfilled the inclusion criteria were included consecutively until the required sample size was reached.

\section{Variables}

Bacterial causes of tonsillitis was the dependent variable while demographic variables (child's age, mother's age, father's age, gender, residence, maternal education, paternal education, and parental occupation), clinical related variables (history of tonsillitis, current type of tonsillitis, number of previous tonsillitis, body temperature, sore throat, swollen tonsils, headache, swollen lymph nodes, difficulty in swallowing, white exudates on the throat, weight loss, tonsillar structural change and history of drug use) were the independent variables. Moreover, patient related variables such as history of contact with someone who had cough, type of breast feeding, attending day care and school, living in overcrowded environment and exposure to wood biofuel were taken as the independent variables.

\section{Data Collection}

A structured questionnaire was used to collect data on demographic characteristics, clinical profiles and other variables. Demographic and other related profiles of the children were collected with face - to - face interviews of their caregivers. Clinical data of children with tonsillitis were screened by the attending pediatricians.

Page $3 / 22$ 


\section{Throat Swab Sample Collection and processing}

Throat swabs were taken by the attending pediatricians from each patient using a sterile cotton swab. Visible exudates or hyperemic areas on tonsillar walls were swabbed with a sterile cotton swab while the tongue depressed by a wooden spatula when necessary.

All the swab samples were immediately transported to the Microbiology Department of HGH using Amie's transport medium (Oxoid, England). Swabs were simultaneously plated onto Blood Agar (BA), Chocolate Agar (CA), and MacConkey (MAC) Agar and incubated for $48 \mathrm{~h}$ at $37^{\circ} \mathrm{C}$. Chocolate Agar was incubated in a candle jar to get $5 \% \mathrm{CO}_{2}$ while BA and MAC were incubated at a normal atmosphere.

\section{Identification of bacterial isolates}

Pure colonies of the bacterial isolates were identified in to species following standard enzymatic and biochemical tests [6]. Small colonies, Gram positive cocci arranged in chain, forming complete hemolysis on BA and both coagulase and catalase negative were taken as S. pyogenes isolates. S. pneumoniae isolates were identified by Gram positive alpha-hemolytic small colonies on BA and were susceptible to optochin. S. aureus isolates were identified by Gram positive cluster forming glistering golden yellow colonies on BA and Mannitol Salt Agar (MSA) which were coagulase, catalase and oxidase positive. Moraxella catarrhalis were identified by large kidney shaped diplococci Gram negative grey to white hemispheric colonies on BA with both oxidase and catalase positive. $K$. pneumoniae and $P$. aeruginosa isolates were identified by standard manual biochemical tests.

\section{Antimicrobial susceptibility testing}

Susceptibilities of all the identified bacterial isolates to different antimicrobials were performed according to the criteria of Clinical and Laboratory Standards Institute (CLSI, 2019) using the Kirby-Bauer disc diffusion method on Mueller-Hinton Agar (MHA) (Himedia, India). The following drug discs were tested: ampicillin $(10 \mu \mathrm{g})$, amoxicillin-clavulanic acid $(20 / 10 \mu \mathrm{g})$, gentamicin $(10 \mu \mathrm{g})$, clarithromycin $(15 \mu \mathrm{g})$, erythromycin $(15 \mu \mathrm{g})$, vancomycin $(30 \mu \mathrm{g})$, ofloxacin $(5 \mu \mathrm{g})$ and ciprofloxacin $(5 \mu \mathrm{g})$. A loop full of culture was taken from a pure culture colony and transferred to a tube containing $5 \mathrm{ml}$ of normal saline and mixed gently until it forms a homogenous suspension. The turbidity of the suspension was then adjusted to the turbidity of McFarland 0.5 (which carries $10^{8}$ $\mathrm{CFU} / \mathrm{ml}$ ) and was swabbed on a dry surface of MHA plate $(150 \mathrm{~mm})$ using a sterile cotton swab. Antibiotic discs were dispensed using a single disc dispenser. Plates were then incubated for $24 \mathrm{~h}$ at $37^{\circ} \mathrm{C}$. Diameters of the zone of inhibition around the discs were measured using a digital caliper. The results of the zone of antibiotics were interpreted based on the $2019 \mathrm{CLSI}$ guideline [7]. These antimicrobial drug discs were selected based on the frequent prescriptions of these drugs for the treatment of tonsillitis infection in the study area and using the 2019 CLSI [7].

\section{Detection of Methicillin Resistant Staphylococcus aureus}

Methicillin resistant Staphylococcus aureus was screened using oxacillin disk diffusion suceptibility testing. Pure colonies of Staphylococcus aureus were inoculated on MSA and $30 \mu \mathrm{g}$ of oxacillin discs were impregnated on the plate and incubated for $18 \mathrm{~h}$. The zone of inhibiton was measured by caliper. Measurements from the CLSI 2019 standard was followed as reference for interpretation. Accordingly, a zone of inhibition of $\leq 21 \mathrm{~mm}$ of oxacillin disk against Staphylococcus aureus isolates were considered as mecA positive and reported as methcillin resistant while if the zone of inhibition of oxacillin disc towards Staphylococcus aureus is $\geq 25 \mathrm{~mm}$, it was considered as mecA negative, and reported as methicillin sensitive[7].

\section{Quality Control}

Specimens were collected properly following standard bacteriological procedures. In order to prevent contamination, all the throat swab specimens were analyzed within two hours of collection. Culture media were checked for sterility. The media were tested every time after preparation for sterility checking by incubating a plate of each medium overnight in a different incubator than one used for culture. The performance of all the prepared culture media were checked by using American Type Culture Collection (ATCC) standard reference strains (S. aureus ATCC 29213, S. penumoniae ATCC 49618, and P. aeruginosa ATCC 27853).

\section{Data analysis}

Data were coded and analyzed using IBM SPSS statistics for windows version 25 (IBM Corp, Armonk, NY, USA). Univariate analysis was made to generate summary values for the most important variables. Logistic regression analysis was made to determine the association between dependent and independent variables. The generated data were compiled with frequency tables and other 
statistical summary measures. Stepwise logistic regression model was used to find factors associated with culture positive bacterial tonsillitis and statistical significance was set at $p<0.05$.

\section{Ethical Considerations}

An ethical approval letter was obtained from the Institutional Review Board (IRB) of College of Medicine and Health Science (CMHS), Bahir Dar University. A permission letter was obtained from the Ministry of Health, Somaliland, and Hargeisa Group of Hospital (HGH). Following well-versed about the purpose and importance of the study, written informed consent was obtained from children parents/guardians before collecting data. Information obtained during this study was kept confidential and used only for the study purpose. Bacteriological positive results were submitted to health pediatricians.

\section{Results}

\section{Characteristics of the study participants}

A total of 374 children within five years of age with tonsillitis took part with a response rate of $97.4 \%$. Among them, 200 (53.5\%) were males. Most (81.6\%) of the children were urban dwellers. The age range of children was 2 to 5 years. Majority (3.7\%) of the children were five years old (mean $=4.1$, median $=4)$. The children mother's age ranged from $20-45$ years. Most $(69 \%)$ of the parents were employee (Table 1). 
Table 1

Socio-demographic characteristics and culture confirmed bacterial tonsillitis among children within five years of age at Hargeisa Group of Hospital

\begin{tabular}{|c|c|c|c|c|c|}
\hline \multirow[t]{3}{*}{ Variables } & \multirow{3}{*}{$\begin{array}{l}\text { Total } \\
\text { N (\%) }\end{array}$} & \multicolumn{2}{|c|}{ Culture results } & \multirow[t]{3}{*}{ COR $(95 \% \mathrm{Cl})$} & \multirow[t]{3}{*}{ P-value } \\
\hline & & Positive & Negative & & \\
\hline & & $\mathbf{N}(\%)$ & $\mathbf{N}(\%)$ & & \\
\hline \multicolumn{5}{|l|}{ Children age (years) } & \multirow[t]{5}{*}{0.199} \\
\hline 2 & $2(0.5)$ & $2(100)$ & 0 & NA & \\
\hline 3 & $110(29.4)$ & $36(32.7)$ & $74(67.3)$ & & \\
\hline 4 & $121(32.4)$ & $40(33.1)$ & $81(66.9)$ & & \\
\hline 5 & $141(37.7)$ & $42(29.8)$ & $99(70.2)$ & & \\
\hline \multicolumn{6}{|l|}{ Gender } \\
\hline Male & $200(53.5)$ & $73(36.5)$ & $127(63.5)$ & $0.64(0.41-1.00)$ & \multirow[t]{2}{*}{0.05} \\
\hline Female & $174(46.5)$ & $47(27)$ & $127(73)$ & & \\
\hline \multicolumn{6}{|l|}{ Residence } \\
\hline Rural & $69(18.4)$ & $19(27.5)$ & $50(72.5)$ & & \multirow[t]{2}{*}{0.37} \\
\hline Urban & $305(81.6)$ & $101(33.1)$ & $204(66.9)$ & $0.78(0.43-1.37)$ & \\
\hline \multicolumn{6}{|l|}{ Father's education } \\
\hline Not able to read and write & $55(14.7)$ & $18(32.7)$ & $37(67.3)$ & $3.46(0.67=17.72)$ & \multirow[t]{5}{*}{0.002} \\
\hline Able to read and write & $56(15)$ & $29(51.8)$ & $27(48.2)$ & $2.53(0.46-13.79)$ & \\
\hline Primary school & $81(21.7)$ & $19(23.5)$ & $62(76.5)$ & $1.01(0.20-5.57)$ & \\
\hline High school & $82(21.9)$ & $30(36.6)$ & $52(63.4)$ & $0.9(0.16-5.04)$ & \\
\hline Higher education & $100(26.7)$ & $24(24)$ & $76(76)$ & & \\
\hline \multicolumn{6}{|l|}{ Mother's education } \\
\hline Not able to read and write & $186(49.7)$ & $78(41.9)$ & $108(58.1)$ & NA & \multirow[t]{5}{*}{0.001} \\
\hline Able to read and write & $105(28.1)$ & $28(26.7)$ & 77 (73.3) & & \\
\hline Primary school & $36(9.6)$ & $7(19.4)$ & $29(80.6)$ & & \\
\hline High school & $30(8)$ & $6(20)$ & $24(80)$ & & \\
\hline Higher education & $17(4.5)$ & $1(5.9)$ & $16(94.1)$ & & \\
\hline \multicolumn{6}{|l|}{ Parental occupation } \\
\hline Unemployed & $101(31)$ & $26(22.4)$ & $90(77.6)$ & $1.98(1.19-3.29)$ & \multirow[t]{2}{*}{0.020} \\
\hline Employed & $258(69)$ & $94(36.4)$ & $164(63.6)$ & & \\
\hline \multicolumn{6}{|l|}{ Mother's age (years) } \\
\hline $20-25$ & $98(26.2)$ & $26(26.5)$ & $72(73.5)$ & NA & 0.14 \\
\hline $26-30$ & 169 (45.2) & $59(34.9)$ & $110(65.1)$ & & \\
\hline $31-35$ & $90(24.1)$ & $27(30)$ & $63(70)$ & & \\
\hline $36-40$ & $15(4)$ & $6(40)$ & $9(60)$ & & \\
\hline
\end{tabular}

Key: NA: Not applicable 


\begin{tabular}{|c|c|c|c|c|c|}
\hline \multirow[t]{3}{*}{ Variables } & \multirow{3}{*}{$\begin{array}{l}\text { Total } \\
\mathbf{N}(\%)\end{array}$} & \multicolumn{2}{|c|}{ Culture results } & \multirow[t]{3}{*}{ COR (95\% Cl) } & \multirow[t]{3}{*}{ P-value } \\
\hline & & Positive & Negative & & \\
\hline & & $\mathbf{N}(\%)$ & $\mathbf{N}(\%)$ & & \\
\hline $41-45$ & $2(0.5)$ & 2 & 0 & & \\
\hline Total & $374(100)$ & $120(32.1)$ & 254 (77.9) & & \\
\hline
\end{tabular}

\section{Bacterial tonsillitis}

Overall, $120(32.1 \%)$ of the children had culture confirmed bacterial tonsillitis. The proportion of bacterial tonsillitis was higher in males $76(36.5 \%)$ than females $47(27 \%)$. It was higher in urban $101(33.1 \%)$ than rural $19(27.5 \%)$ residents. The percentage of bacterial tonsillitis was higher (41.9\%) in children from mother's unable to read and write than other groups (5.9-26.7\%). Children from fathers who had higher educational attainment had lowest percentage of bacterial tonsillitis compared to others (Table 1).

\section{Bacterial tonsillitis and clinical profiles}

Table 2 depicts the bacterial tonsillitis with clinical profiles among children within five years of age. From the total, $172(46 \%)$ of children had a history of tonsillitis. The majority of children had presented with acute tonsillitis (54\%) and sore throat (91.7\%). Swollen tonsils were presented in $98.1 \%$ of children. On the other hand, cervical lymphadenopathy was presented in 151 (40.4\%) of children. Moreover, 147 (39.3\%) and 69 (18.4\%) of children had difficulty of swallowing and white exudates, respectively (Table 2). 
Table 2

Bacterial tonsilitis and clinical profiles of children with tonsillitis at Hargeisa Group of Hospital

\begin{tabular}{|c|c|c|c|c|c|}
\hline \multirow[t]{3}{*}{ Variables } & \multicolumn{3}{|c|}{ Culture results } & \multicolumn{2}{|c|}{ COR (95\% Cl) P-value } \\
\hline & \multirow{2}{*}{$\begin{array}{l}\text { Total } \\
\text { N (\%) }\end{array}$} & \multirow{2}{*}{$\begin{array}{l}\text { Positive } \\
\text { N (\%) }\end{array}$} & \multirow{2}{*}{$\begin{array}{l}\text { Negative } \\
\mathrm{N}(\%)\end{array}$} & & \\
\hline & & & & & \\
\hline \multicolumn{6}{|c|}{ History of tonsillitis } \\
\hline Yes & $172(46)$ & $95(55.2)$ & $77(44.8)$ & $8.72(5.22-14.63)$ & $<0.001$ \\
\hline No & $202(54)$ & $25(12.4)$ & 177 (87.6) & & \\
\hline \multicolumn{6}{|c|}{ Number of previous tonsillitis } \\
\hline None & $202(54)$ & $26(12.9)$ & $176(87.1)$ & & \\
\hline One & $15(4)$ & $9(60)$ & $6(40)$ & $8(4.63-13.82)$ & $<0.001$ \\
\hline Two & $24(6.4)$ & $14(58.3)$ & $10(41.7)$ & $1.38(0.44-4.35)$ & 0.58 \\
\hline Three & $13(3.5)$ & $6(46.2)$ & $7(58.3)$ & $0.84(0.35-2.1)$ & 0.71 \\
\hline$\geq$ Four & $120(32.1)$ & $65(54.2)$ & $55(45.8)$ & $0.79(0.26-2.35)$ & 0.67 \\
\hline \multicolumn{6}{|c|}{ Type of tonsillitis } \\
\hline Acute & $202(54)$ & $25(12.4)$ & 177 (87.6) & & \\
\hline Chronic & $52(13.9)$ & $30(57.7)$ & $22(42.3)$ & $0.11(0.68-0.19)$ & $<0.001$ \\
\hline Recurrent & $120(32.1)$ & $65(54.2)$ & $55(45.8)$ & & \\
\hline \multicolumn{6}{|c|}{ Weight loss } \\
\hline Yes & $143(38.2)$ & $67(46.9)$ & $76(53.1)$ & $2.96(1.89-4.64)$ & $<0.001$ \\
\hline No & $231(61.8)$ & $53(22.9)$ & $178(77.1)$ & & \\
\hline \multicolumn{6}{|c|}{ Tonsillar structural change } \\
\hline Yes & $52(13.9)$ & $30(57.7)$ & $22(42.3)$ & $3.52(1.93-6.42)$ & $<0.001$ \\
\hline No & $322(86.1)$ & $90(28)$ & $232(72)$ & & \\
\hline \multicolumn{6}{|c|}{ Body temperature } \\
\hline $37 C^{\circ}$ & $42(11.2)$ & $12(28.6)$ & $30(71.4)$ & $1.21(0.59-)$ & 0.61 \\
\hline$>38^{\circ} \mathrm{C}$ & $301(88.8)$ & $108(32.5)$ & $224(67.5)$ & $1.21(0.59-)$ & \\
\hline \multicolumn{6}{|c|}{ Sore throat } \\
\hline Yes & $343(91.7)$ & $111(32.4)$ & 232 (63.6) & $0.86(0.38-1.92)$ & 0.704 \\
\hline No & $31(8.3)$ & $9(29)$ & $22(71)$ & & \\
\hline \multicolumn{6}{|c|}{ Swollen tonsils } \\
\hline Yes & $367(98.1)$ & 119 (32.4) & $248(67.6)$ & $0.35(0.04-2.92)$ & 0.308 \\
\hline No & $7(1.9)$ & $1(14.3)$ & $6(85.7)$ & & \\
\hline \multicolumn{6}{|l|}{ Headache } \\
\hline Yes & $105(28.1)$ & 33 (31.4) & $72(68.6)$ & $1.04(0.64-1.69)$ & 0.865 \\
\hline No & $269(71.9)$ & 87 (32.3) & $182(67.7)$ & & \\
\hline
\end{tabular}




\begin{tabular}{|c|c|c|c|c|c|}
\hline \multirow[t]{3}{*}{ Variables } & \multicolumn{3}{|c|}{ Culture results } & \multicolumn{2}{|c|}{ COR (95\% Cl) P-value } \\
\hline & \multirow{2}{*}{$\begin{array}{l}\text { Total } \\
\mathrm{N}(\%)\end{array}$} & \multirow{2}{*}{$\begin{array}{l}\text { Positive } \\
\text { N (\%) }\end{array}$} & \multirow{2}{*}{$\begin{array}{l}\text { Negative } \\
\mathrm{N}(\%)\end{array}$} & & \\
\hline & & & & & \\
\hline Yes & $151(40.4)$ & $53(35.1)$ & $98(64.9)$ & $1.26(0.81-1.96)$ & 0.304 \\
\hline No & $223(59.6)$ & $67(30)$ & $156(70)$ & & \\
\hline \multicolumn{6}{|c|}{ White exudates } \\
\hline Yes & $69(18.4)$ & $19(27.5)$ & $50(72.5)$ & $0.77(0.43-1.37)$ & \multirow[t]{2}{*}{0.370} \\
\hline No & $305(81.6)$ & $101(33.1)$ & $204(66.9)$ & & \\
\hline \multicolumn{6}{|c|}{ Difficulty of swallowing } \\
\hline Yes & 147 (39.3) & $27(18.4)$ & $120(81.6)$ & $0.33(0.19-0.53)$ & \multirow[t]{2}{*}{$<0.001$} \\
\hline No & $227(60.7)$ & $93(41)$ & $134(59)$ & & \\
\hline
\end{tabular}

The percentage of bacterial tonsillitis was higher among children with a history of tonsillitis $(55.2 \%)$ than others $(12.4 \%)$. The percentage of tonsillitis was the highest (55.7\%) in children with symptoms of chronic tonsillitis. Moreover, the percentage of bacterial tonsillitis was higher in children with tonsillar structural change $(57.7 \%)$ than those without (28\%). Children with swollen tonsils had higher percentage of culture confirmed tonsillitis (32.4\%) than those without swollen tonsils (14.3\%). The proportion of culture confirmed bacterial tonsillitis was higher among children who had weight loss (46.9\%) than the counters (22.9\%) (Table 2).

\section{Bacterial tonsillitis in relation to other variables}

Overall, 96 (25.7\%) and $228(61 \%)$ of children were exclusively breastfed and had history of contact with coughing patients, respectively. On the other hand, $86(23 \%)$ and $282(75.4 \%)$ of children were daycare center attendees and school attendees, respectively. Most of the children lived in a crowded house (71.7\%) and $88.5 \%$ had exposure to biofuel (Table 3 ). 
Table 3

Distribution of bacterial tonsillitis and other explanatory variables of children within five years of age with symptoms of tonsillitis at Hargeisa Group of hospital.

\begin{tabular}{|c|c|c|c|c|c|}
\hline \multirow[t]{3}{*}{ Variables } & \multicolumn{3}{|c|}{ Culture result } & \multicolumn{2}{|c|}{ COR (95\% Cl) P-value } \\
\hline & \multirow{2}{*}{$\begin{array}{l}\text { Total } \\
\text { N (\%) }\end{array}$} & \multirow[t]{2}{*}{ Positive N (\%) } & \multirow[t]{2}{*}{ Negative N (\%) } & & \\
\hline & & & & & \\
\hline \multicolumn{6}{|c|}{ Contact with cough patients } \\
\hline Yes & $228(61)$ & 85 (37.3) & $143(62.7)$ & $1.89(1.18-3.00)$ & \multirow[t]{2}{*}{0.008} \\
\hline No & $146(39)$ & $35(24)$ & $111(76)$ & & \\
\hline \multicolumn{6}{|c|}{ Breast feeding } \\
\hline Mixed & $278(74.3)$ & $91(32.7)$ & 187 (67.3) & $1.12(0.68-1.86)$ & \multirow[t]{2}{*}{0.65} \\
\hline Exclusive & $96(25.7)$ & $29(30.2)$ & $67(69.8)$ & & \\
\hline \multicolumn{6}{|c|}{ Day care center attendee } \\
\hline Yes & $86(23)$ & $34(39.5)$ & $52(60.5)$ & $1.54(0.93-2.53)$ & \multirow[t]{2}{*}{0.092} \\
\hline No & $288(77)$ & $86(29.9)$ & $202(70.1)$ & & \\
\hline \multicolumn{6}{|c|}{ Living in over crowded house } \\
\hline Yes & $268(71.7)$ & $76(28.4)$ & $192(71.6)$ & $0.56(0.35-0.89)$ & \multirow[t]{2}{*}{0.014} \\
\hline No & $106(28.3)$ & $44(41.5)$ & $62(58.5)$ & & \\
\hline \multicolumn{6}{|c|}{ Exposure to wood biofuel } \\
\hline Yes & $331(88.5)$ & $117(35.3)$ & $214(64.7)$ & $7.29(2.21-24.1)$ & 0.001 \\
\hline No & $43(11.5)$ & $3(7)$ & $40(93)$ & & \\
\hline \multicolumn{6}{|c|}{ School attendee } \\
\hline Yes & $92(24.6)$ & $48(52.2)$ & $44(47.8)$ & $0.31(0.19-0.51)$ & $<0.001$ \\
\hline No & $282(75.4)$ & $72(25.5)$ & $210(74.5)$ & & \\
\hline Total & $374(100)$ & $120(32.1)$ & 254 (67.9) & & \\
\hline
\end{tabular}

The proportion of culture confirmed bacterial tonsillitis was higher among children who had history of contact with coughing patients $(61 \%)$ than the counters (24\%). Daycare center attendee children had a higher (39.5\%) percentage of bacterial tonsillitis than others (29.9\%). Moreover, school attending children had a higher (52.2\%) percentage of bacterial tonsillitis than the counterparts (25.5\%). The proportion of bacterial tonsillitis was higher among children who had exposure to biofuel (35.3\%) than others (7\%) (Table 3).

\section{Distribution of bacteria isolates}

A total of $143(32 \%)$ bacterial pathogens were isolated from 120 culture positive samples. The most frequent isolate was $S$. pyogenes 78 (55\%) followed by S. aureus 42 (29\%) and S. pneumoniae 10 (7\%) (Fig. 1). Among the 120 children with confirmed bacterial tonsillitis, $23(19.2 \%)$ had mixed infections. S. pyogenes and S. aureus, S. pneumoniae and S. aureus and S. aureus and M. catarrhalis were the most common mixed isolates with a proportion of $10(8.3 \%), 4(3.3 \%)$ and $4(3.3 \%)$, respectively (Table 4$)$. 
Table 4

Distribution of mixed isolates and MRSA from the

total culture confirmed bacterial tonsilities $(n=$

120) among children within five years of age at Hargeisa Group of Hospital

\begin{tabular}{|ll|}
\hline Type of mixed isolates & $\begin{array}{l}\text { Frequency } \\
\text { (\%) }\end{array}$ \\
\hline S. pyogenes + S. aureus & $10(8.3)$ \\
\hline S. pyogenes + P. aeruginosa & $3(2.5)$ \\
\hline S. pneumoniae + S. aureus & $4(3.3)$ \\
\hline S. pneumoniae + K. pneumoniae & $2(1.7)$ \\
\hline S. aureus + M. catarrhalis & $4(3.3)$ \\
\hline Total & $23(19.2)$ \\
\hline MRSA & $19(15.8 \%)$ \\
\hline Key: MRSA: Methicillin resistant S. aureus \\
\hline
\end{tabular}

\section{Antibiotic resistance profiles of bacterial isolates}

Overall, 131 (91.6\%) bacterial isolates were resistant to ampicillin. Relatively, higher resistance percentages were found against gentamicin (41.3\%), ofloxacin (34.3\%) and clarithromycin (32.2\%). S. pyogenes, S. aureus, S. pneumoniae and M. catarrhalis isolates revealed an overall resistance of $33.5 \%, 37.2 \%, 28.8 \%$ and $50 \%$, respectively. S. pyogenes revealed resistant to ampicillin (94.9\%), ofloxacin (43.6\%), and gentamicin (42.3\%). S. aureus isolates showed resistance to ampicillin (83.8\%) clarithromycin (38.1\%), and ciprofloxacin (35.7\%). The percentage of $S$. pneumoniae isolates resistance to ampicillin, gentamicin, clarithromycin and erythromycin was $100 \%, 60 \%, 30 \%$, and $30 \%$, respectively. Klebsiella pneumoniae isolates were resistant to ampicillin (83.3\%) and erythromycin (66.7\%). All P. aeruginosa isolates were resistant to ciprofloxacin and ampicillin (Table 5). 
Table 5

Antibiotic resistance profile of bacterial isolates from children under the age of five with tonsillitis at Hargeisa Group of Hospital

\begin{tabular}{|c|c|c|c|c|c|c|c|c|c|c|c|c|c|c|}
\hline \multirow[t]{2}{*}{$\begin{array}{l}\text { Antibiotics } \\
\text { tested }\end{array}$} & \multicolumn{2}{|c|}{$\begin{array}{l}\text { S. pyogenes } \\
(n=78)\end{array}$} & \multicolumn{2}{|c|}{$\begin{array}{l}\text { S. aureus } \\
(n=42)\end{array}$} & \multicolumn{2}{|c|}{$\begin{array}{l}S . \\
\text { pneumoniae } \\
(n=10)\end{array}$} & \multicolumn{2}{|c|}{$\begin{array}{l}\text { M. } \\
\text { catarrhalis } \\
(n=4)\end{array}$} & \multicolumn{2}{|c|}{$\begin{array}{l}P . \\
\text { aeruginosa } \\
(n=3)\end{array}$} & \multicolumn{2}{|c|}{$\begin{array}{l}\text { K. } \\
\text { pneumoniae } \\
(n=6)\end{array}$} & \multicolumn{2}{|c|}{$\begin{array}{l}\text { Total } \\
(n=143)\end{array}$} \\
\hline & $\# \mathrm{~T}$ & $\mathrm{R} \%$ & $\# \mathrm{~T}$ & $R \%$ & \#T & $\mathrm{R} \%$ & \#T & $\mathrm{R} \%$ & $\# \mathrm{~T}$ & $\mathrm{R} \%$ & \#T & $\mathrm{R} \%$ & \#T & $\mathrm{R} \%$ \\
\hline $\begin{array}{l}\text { Amoxicillin- } \\
\text { clavulanic } \\
\text { acid }\end{array}$ & 78 & $\begin{array}{l}6 \\
(7.7)\end{array}$ & 42 & $\begin{array}{l}10 \\
(23.8)\end{array}$ & 10 & 0 & 4 & $\begin{array}{l}2 \\
(50)\end{array}$ & 3 & $\begin{array}{l}1 \\
(33.3)\end{array}$ & 6 & $\begin{array}{l}2 \\
(33.3)\end{array}$ & 143 & $\begin{array}{l}21 \\
(14.7)\end{array}$ \\
\hline Ciprofloxacin & 78 & $\begin{array}{l}8 \\
(10.3)\end{array}$ & 42 & $\begin{array}{l}15 \\
(35.7)\end{array}$ & 10 & 0 & 4 & $\begin{array}{l}2 \\
(50)\end{array}$ & 3 & $\begin{array}{l}3 \\
(100)\end{array}$ & 6 & $\begin{array}{l}2 \\
(33.3)\end{array}$ & 143 & $\begin{array}{l}30 \\
(21)\end{array}$ \\
\hline Clarithromycin & 78 & $\begin{array}{l}22 \\
(28.2)\end{array}$ & 42 & $\begin{array}{l}16 \\
(38.1)\end{array}$ & 10 & $3(30)$ & 4 & $\begin{array}{l}2 \\
(50)\end{array}$ & 3 & $\begin{array}{l}1 \\
(33.3)\end{array}$ & 6 & $\begin{array}{l}2 \\
(33.3)\end{array}$ & 143 & $\begin{array}{l}46 \\
(32.2)\end{array}$ \\
\hline Gentamicin & 78 & $\begin{array}{l}33 \\
(42.3)\end{array}$ & 42 & $\begin{array}{l}13 \\
(31)\end{array}$ & 10 & $6(60)$ & 4 & $\begin{array}{l}3 \\
(75)\end{array}$ & 3 & $\begin{array}{l}2 \\
(66.7)\end{array}$ & 6 & $\begin{array}{l}2 \\
(33.3)\end{array}$ & 143 & $\begin{array}{l}59 \\
(41.3)\end{array}$ \\
\hline Vancomycin & 78 & $\begin{array}{l}10 \\
(12.8)\end{array}$ & 42 & $\begin{array}{l}14 \\
(33.3)\end{array}$ & 10 & 0 & 4 & 0 & 3 & $\begin{array}{l}1 \\
(33.3)\end{array}$ & 6 & $\begin{array}{l}2 \\
(33.3)\end{array}$ & 143 & $\begin{array}{l}27 \\
(18.9)\end{array}$ \\
\hline Ofloxacin & 78 & $\begin{array}{l}34 \\
(43.6)\end{array}$ & 42 & $\begin{array}{l}11 \\
(26.2)\end{array}$ & 10 & $1(10)$ & 4 & 0 & 3 & $\begin{array}{l}2 \\
(66.7)\end{array}$ & 6 & $\begin{array}{l}1 \\
(16.7)\end{array}$ & 143 & $\begin{array}{l}49 \\
(34.3)\end{array}$ \\
\hline Erythromycin & 78 & $\begin{array}{l}22 \\
(28.2)\end{array}$ & 42 & $\begin{array}{l}11 \\
(26.2)\end{array}$ & 10 & $3(30)$ & 4 & $\begin{array}{l}3 \\
(75)\end{array}$ & 3 & $\begin{array}{l}2 \\
(66.7)\end{array}$ & 6 & $\begin{array}{l}4 \\
(66.7)\end{array}$ & 143 & $\begin{array}{l}45 \\
(31.5)\end{array}$ \\
\hline Ampicillin & 78 & $\begin{array}{l}74 \\
(94.9)\end{array}$ & 42 & $\begin{array}{l}35 \\
(83.3)\end{array}$ & 10 & $10(100)$ & 4 & $\begin{array}{l}4 \\
(100)\end{array}$ & 3 & $\begin{array}{l}3 \\
(100)\end{array}$ & 6 & $\begin{array}{l}5 \\
(83.3)\end{array}$ & 143 & $\begin{array}{l}131 \\
(91.6)\end{array}$ \\
\hline Total & 624 & $\begin{array}{l}209 \\
(33.5)\end{array}$ & 336 & $\begin{array}{l}125 \\
(37.2)\end{array}$ & 80 & $\begin{array}{l}23 \\
(28.8)\end{array}$ & 32 & $\begin{array}{l}16 \\
(50)\end{array}$ & 24 & $\begin{array}{l}15 \\
(62.5)\end{array}$ & 48 & $\begin{array}{l}20 \\
(41.7)\end{array}$ & 1144 & $\begin{array}{l}408 \\
(35.7)\end{array}$ \\
\hline
\end{tabular}

\section{Multiple Drug Resistant (MDR) profiles of bacterial isolates}

Overall, $72(50.4 \%)$ of the bacterial species were MDR and $52.6 \%$ of $S$. pyogenes were MDR. The MDR profile of $S$. aureus, $S$. pneumoniae and Klebsiella pneumoniae isolates were 18 (42.9\%), 6 (60\%) and $3(50 \%)$, respectively (Table 6).

Table 6

Multidrug resistance profiles of bacterial isolates from children within five years of age with tonsilitis at Hargeisa Group of Hospital.

\begin{tabular}{|c|c|c|c|c|c|c|c|}
\hline \multirow[t]{2}{*}{ Bacterial species } & $\mathbf{R} 1$ & R2 & R3 & R4 & R5 & R6 & Over all MDR \\
\hline & $N(\%)$ & $\mathbf{N}(\%)$ & $N(\%)$ & $\mathbf{N}(\%)$ & $N(\%)$ & $\mathbf{N}(\%)$ & $N(\%)$ \\
\hline S. pyogenes (78) & $16(20.5)$ & $21(26.9)$ & $24(30.3)$ & $14(17.9)$ & $3(3.8)$ & 0 & $41(52.6)$ \\
\hline S. aureus (42) & $8(19)$ & $16(38)$ & $2(4.8)$ & 7 (16.7) & $5(11.9)$ & $4(9.5)$ & $18(42.9)$ \\
\hline S. pneumoniae (10) & $4(40)$ & & $5(50)$ & $1(10)$ & 0 & 0 & $6(60)$ \\
\hline K. pneumoniae (6) & $1(16.7)$ & $2(33.3)$ & $1(16.7)$ & $2(33.3)$ & 0 & 0 & $3(50)$ \\
\hline M. catarrhalis (4) & $1(25)$ & $2(50)$ & $1(25)$ & 0 & 0 & 0 & $1(25)$ \\
\hline P. aeruginosa (3) & 0 & 0 & $2(66.7)$ & $1(33.3)$ & 0 & 0 & $3(100)$ \\
\hline Total (143) & $30(20.9)$ & $41(28.7)$ & $35(24.5)$ & $25(17.5)$ & $8(5.6)$ & $4(2.8)$ & $72(50.4)$ \\
\hline
\end{tabular}


Based on multivariable analysis, bacterial tonsillitis was significantly associated with difficulty of swallowing $(A O R=6.99, \mathrm{Cl}=3.56-$ 13.13), weight loss $(A O R=0.33, \mathrm{Cl}=0.186-0.597)$, attending school $(A O R=2.98, C l=1.64-5.42)$, history of tonsillitis $(A O R=0.12, \mathrm{Cl}$ $=0.06-0.21)$ and exposure to biofuel $(A O R=0.19, \mathrm{Cl}=0.04-0.84)$. Children who had difficulty of swallowing were 7 times more likely to become culture positive for bacterial tonsillitis, compared to children who did not have difficulty of swallowing. Likewise, school attending children were 3 times more likely to have confirmed bacterial tonsillitis compared to non-attendees. Children with history of tonsillitis were more likely to had bacterial tonsillitis than those without history of tonsillitis. Similarly, children who had weight loss and exposure to biofuel were more likely to become culture confirmed tonsillitis compared to those who did not have weight loss and exposure to biofuel (Table 7). 
Table 7

Multivariable analysis of factors associated with bacterial tonsillitis among children under the age of five years at $\mathrm{HGH}$

\begin{tabular}{|c|c|c|c|c|}
\hline Variables & COR $(95 \% \mathrm{Cl})$ & P-value & AOR $(95 \% \mathrm{Cl})$ & P-value \\
\hline \multicolumn{5}{|l|}{ Gender } \\
\hline Male & $0.64(0.41-1.00)$ & 0.05 & $0.68(0.38-1.19)$ & 0.18 \\
\hline \multicolumn{5}{|l|}{ Female } \\
\hline \multicolumn{5}{|c|}{ History of tonsillitis } \\
\hline Yes & $0.11(0.68-0.19)$ & $<0.001$ & $01.2(0.06-0.21)$ & $<0.001$ \\
\hline \multicolumn{5}{|l|}{ No } \\
\hline \multicolumn{5}{|c|}{ Type of tonsillitis } \\
\hline \multicolumn{5}{|l|}{ Acute } \\
\hline Chronic & $8.37(4.82-14.53)$ & $<0.001$ & & \\
\hline Recurrent & $0.87(0.45-1.67)$ & 0.67 & $0.45(0.01-17.2)$ & 0.67 \\
\hline \multicolumn{5}{|c|}{ Parental occupation } \\
\hline Unemployed & $1.98(1.19-3.29)$ & 0.008 & $1.68(0.89-3.18)$ & 0.11 \\
\hline \multicolumn{5}{|l|}{ Employed } \\
\hline \multicolumn{5}{|c|}{ Contact with cough } \\
\hline Yes & $1.89(1.18-3.0)$ & 0.008 & $0.71(0.37-1.33)$ & 0.29 \\
\hline \multicolumn{5}{|l|}{ No } \\
\hline \multicolumn{5}{|c|}{ Tonsillar stricture } \\
\hline Yes & $3.52(1.93-6.42)$ & $<0.001$ & $2.1(0.05-80.7)$ & 0.69 \\
\hline \multicolumn{5}{|l|}{ No } \\
\hline \multicolumn{5}{|l|}{ Over crowded } \\
\hline Yes & $0.56(0.35-0.89)$ & 0.02 & $0.93(0.45-1.91)$ & 0.93 \\
\hline \multicolumn{5}{|l|}{ No } \\
\hline \multicolumn{5}{|c|}{ Difficulty of swallowing } \\
\hline Yes & $0.32(0.198-0.53)$ & $<0.001$ & 6.99 (3.56-13.73) & $<0.001$ \\
\hline \multicolumn{5}{|l|}{ No } \\
\hline \multicolumn{5}{|l|}{ Weight loss } \\
\hline Yes & $2.96(1.89-4.64)$ & $<0.001$ & $0.33(0.186-0.597)$ & $<0.001$ \\
\hline \multicolumn{5}{|l|}{ No } \\
\hline \multicolumn{5}{|c|}{ Attending school } \\
\hline Yes & $0.31(0.19-0.51)$ & $<0.001$ & $2.98(1.64-5.42)$ & $<0.001$ \\
\hline \multicolumn{5}{|l|}{ No } \\
\hline \multicolumn{5}{|c|}{ Attending day care center } \\
\hline Yes & $1.54(0.93-2.53)$ & 0.09 & $1(0.44-2.34)$ & 0.97 \\
\hline
\end{tabular}

Page $14 / 22$ 


\begin{tabular}{|lllll|}
\hline Variables & COR $(95 \% \mathrm{Cl})$ & P-value & AOR $(95 \% \mathrm{Cl})$ & P-value \\
\hline Wood biofuel & & & & \\
\hline Yes & $7.29(2.21-24.1)$ & 0.001 & $0.19(0.04-0.84)$ & 0.029 \\
\hline No & & & & \\
\hline
\end{tabular}

\section{Discussion}

Tonsillitis has considerably a negative impact on the patients' quality of life and has a significant burden on public health. Untreated childhood tonsillitis can leads to peritonsillar abscess, tonsillar stones, and rheumatic fever. Therefore, identification and antimicrobial susceptibility of bacterial causes of tonsillitis is essential to curtail for the treatment of tonsillitis. However, patients with tonsillitis managed empirically in health care's settings of Somaliland. Therefore, this study presents the first report of the prevalence of culture confirmed bacterial tonsillitis and the antimicrobial resistance profiles of isolates in $\mathrm{HGH}$.

In this study, $32.1 \%$ of children within five years of age had culture confirmed bacterial causes of tonsillitis. Due to the lack of previous data in Somaliland, comparison of countrywide results was not possible. However, the prevailing magnitude of tonsillitis is higher than similar studies with a prevalence of $11.3 \%$ in Ethiopia [8], 20.6\% in Tanzania [1], 21.6\% in Norway [9], and 19\% in Bangladesh [10]. On the other hand, the existing prevalence of bacterial causes of tonsillitis from this study was lower than studies done in the United Kingdom (79\%) [11], Trinidad (62.5\%) [12], India (72\%) [4], Saudi Arabia (65\%) [13], Benin (73.97\%)[5] and Ethiopia (51\%) [14]. The lower rate of bacterial tonsillitis in the present study compared to other developing countries might be attributed to differences in geography, community living status and hygienic practices, host factor and educational level of the parents.

The prevalence of bacterial causes of tonsillitis in children within five years of age was higher in males than in females which is similar to studies from India [2] and Nigeria[15]. The variations on the percentage of tonsillitis between genders of the children could be due to the fact that males spend more time in outdoor than females. The percentage of tonsillitis was higher among children living in urban than rural areas. This was similar with studies done in India [2], and Ethiopia [14]. This might be due to variation in: encountering infected people, exposure with air pollution from biofuel use, schooling and house crowding.

High rate of bacterial tonsillitis was reported among patients symptomatic for chronic (57.7\%) and recurrent tonsillar infections (51.2\%). These are indications of antimicrobial resistance and tonsillectomy. Regarding the prevalence of bacterial isolates;

Streptococcus pyogenes was the most frequent bacterial isolate from children with tonsillitis in this study and its percentage $(55 \%)$ is similar with studies in Ohio (58\%) [16], Italy (69\%) [17], and Trinidad (82.2\%) [12]. However, it is higher than studies from Egypt (17\%) [18], Iran (20\%)[19], Iraq (29.7\%)[20], India (22.25\%) [21], Saudi Arabia (40\%)[22], and Ethiopia (12.2\%) [23]. This variation might be influenced by climate changes, age and geographical inhabitation of the study participants.

In the present study, Staphylococcus aureus was the second most frequent isolate of bacterial causes of tonsillitis with a rate of $29 \%$. This could be due to the persistence of $S$. aureus in the tonsillar tissues, treatment with antimicrobials and antibiotic resistance. Moreover, $S$. aureus has the potential to form biofilm which results recurrent and chronic infection as well as treatment failure. The isolation of $S$. aureus as the main agent of tonsillitis has been reported by several authors in Ethiopia [16], Brazil (40\%) [24], Trinidad (68.9\%) [12], and Nigeria (32.1\%) [15].

It is a fact that isolation of $S$. pneumoniae indicates the existence of recurrent tonsillitis in children. The percentage ( $7 \%)$ of Streptococcus pneumoniae isolates from children with tonsillitis in the present study is lower than studies done from Poland (14\%) [25], Belgium (21\%) [26], Italy (4\%) [17], and South Ethiopia (62.5\%) [27]. However, it was higher than studies done in the US (3.5\%) [28], Nepal (4\%) [29] and Nigeria (3.3\%) [30]. The percentage (4\%) of Klebsiella pneumoniae causing tonsillitis in this study is higher than a study done in Brazil (1.4\%) [31], but was lower than studies done in Singapore (6.6\%) [32], Indonesia (7\%)[33]. In this study, the prevalence of Moraxella catarrhalis was $3 \%$ which is different from studies done in the USA (22\%) [34], Brazil (28.5\%) [35], Denmark (53\%) [36], Tanzania (90.8\%) [37], and Ethiopia (12.3\%) [14].

In the present study, there is high proportion of mixed infections particularly with $S$. pyogenes and $S$. aureus, S. pneumoniae and $S$. aureus and $S$. pneumoniae and $M$. catarrhalis. These co-infections of the tonsils may contribute to the severe inflammatory process 
and the failure of penicillin and ampicillin therapy which finally results recurrent infection, tonsillectomy, rheumatic fever and other complications $[34,38]$.

In this study, the percentage of Methicillin Resistant S. aureus (MRSA) isolates among children with tonsillitis was $15.8 \%$. This is higher than studies done in Germany (0.8\%) [37], Lahore (5.5\%) [39], Japan (0.8\%) [40], Brazil (3.3\%) [24] and Ethiopia (2.3\%) [41], but lower than studies done in the USA (16\%) [42], Benin (17.95\%) [5] and Uganda (32\%) [43]. The variation between the studies might be due to geographical variations, age and child contact to hospitalized patients who could have contracted the MRSA from hospitals.

The resistance of the isolates to ampicillin was $91.6 \%$ and $14.7 \%$ to the association of amoxicillin and clavulanate. The higher resistance to ampicillin by all of the bacterial isolates might be due to production of beta lactamase enzyme as well as abuse and excessive use of cheap drugs, which can be afforded and administered without a physician's guidance. This is a major concern that limits the use of this common therapeutic option in clinical practice in developing countries. The rate of penicillin resistance is comparable with reports from Nigeria (100\%) [5].

The resistance rate of $94.9 \%$ of $S$. pyogenes to ampicillin is worrisome. As B -lactam antibiotics are the drug of choice for strep throat. The percentage of $S$. pyogenes resistant to gentamicin (42.3\%) and ofloxacin (43.6\%) in the present study was comparable to studies done in Iran (32.2\%) [44]. The resistance of Streptococcus pyogenes to the above drugs might be due to the enzymatic inactivation mediated by aminoglycoside-modifying enzymes (AMEs), and point mutations in the quinolones resistance-determining region (QRDR).

The resistance of S. aureus to ciprofloxacin (35.7\%) in this study, was lower than studies done in Egypt (90.9\%) [45] and Nepal (100\%) [46], but was higher than studies done in Brazil (24.6\%) [24]. On the other hand, the resistance profile of S. pneumoniae to erythromycin (30\%) is similar to a study done in Malaysia, with a rate of (30\%) but different from studies done in China (56\%, 20\%) [47], Lithuania (78.8\%) [48] and Ethiopia (12.4\%) [49].

One of the major worries when determining resistance profiles of isolates is the availability of MDR strains. In this study, half of the bacterial isolates were MDR. This is a series problem for children within five years of age in Somaliland. Children involved in the study area were outpatients and they might have constant contact with other children and their family. Moreover, in the study area there is no routine culture and antimicrobial susceptibility testing and management of children with tonsillitis is empirical. These may results repeated infections of the tonsils, pyogenic meningitis, rheumatic fever, lower respiratory tract infections and difficulty to select the effective antibiotics. Furthermore, existence of MDR isolates demonstrates persistence of the bacteria and possibility of antimicrobial resistance dissemination and recurrence of infection [34].

The percentage of MDR S. pneumoniae (60\%) in this study was higher than studies from Poland (52.9\%) [50], Lithuania (12.5\%) [48] and Vietnam (35\%) [51]. In this study, all isolates of Pseudomonas aeruginosa were MDR (100\%) which is concurrent to a study in Brazil (100\%) [31]. These high proportions of MDR among the isolates might be due to productions of beta-lactamase enzyme by Pseudomonas aeruginosa and production of Penicillin binding proteins in Streptococcus pneumoniae.

In the present study, difficulty of swallowing is one of the predictors of bacterial tonsillitis in children within five years of age. Similar findings were reported in India [2] and Lithuania (48). History of tonsillitis was also a predictor variable in this study which was similar to studies done in Ethiopia [9], and Yemen [52]. These might be due to cohabitations of the tonsils by multiple bacterial isolates as depicted in Table 4 and failure of penicillin and ampicillin therapy.

Weight loss was also another predictor for bacterial tonsillitis in this study in which similar studies were reported in Iran [53], and Germany [54]. Furthermore, attending school was a risk factor for tonsillitis in this study similar to studies done in Uganda [55], and Australia [56]. This might be due to overcrowding during schooling among children where carrier children can easily interact with healthy children.

\section{Limitations Of The Study}

This study provided the first report of data on bacterial causes of tonsillitis and antibiotic resistance profiles of the isolates from children within five years of age with tonsillitis at Hargeisa Group of Hospital. However, the study limited to identifying nonbacterial causes of tonsillitis. 


\section{Conclusions}

High prevalence of tonsillitis with MDR pathogens, MRSA and mixed isolates were found. S. pyogenes followed by $S$. aureus and $S$. pneumoniae were the most frequent isolates. Most of the bacterial isolates were resistant to ampicillin. However, amoxicillinclavulanic acid and ciprofloxacin are the least resisted drugs. Therefore, the result points the significance of culture and antimicrobial susceptibility testing in the diagnosis and treatment of any form of tonsillitis for the selection of the effective antibiotics against several pathogenic agents of tonsillitis and rational use of antimicrobials.

Further investigation to identify nonbacterial causes of tonsillitis, conducting studies covering larger geographical areas to draw the magnitude and topographic variations are needed to control the spread of tonsillitis among children within five years age.

\section{Abbreviations}

AMEs

Aminoglycoside-Modifying Enzymes

AST

Antimicrobial Sensitivity Testing

ATCC

American Type Culture Collection

AOR

Adjusted Odd Ratio

BA

Blood Agar

CA

Chocolate Agar

CDC

Centre for Disease Control

CFU

Colony Forming Unit

CLSI

Clinical and Laboratory Standard Institute

\section{CMHS}

College of Medicine and Health Science

COR

Crude Odd Ratio

ENT

Ear Nose and Throat

$\mathrm{HGH}$

Hargeisa Group of Hospital

IRB

Institutional Review Board

MAC

MacConkey agar

MDR

Multi-Drug Resistant

MHA

Mueller Hinton Agar

MRSA

Methicillin Resistant Staphylococcus aureus

MSA

Manitol Salt Agar

QRDR 
Quinolones Resistance-Determining Region

SPSS

Statistical Software Package of Social Science

\section{Declarations}

\section{Ethical approval and consent to participate}

This study was approved by the institutional review board (IRB) of College of Medicine and Health Science (CMHS), Bahir Dar University and a permission letter was obtained from the Ministry of Health, Somaliland, and Hargeisa Group of Hospitals (HGH). In addition, written informed consent was obtained from parents/guardians of participating children before collecting data. Information obtained from study participants was kept confidential and used only for the purpose of this study. Bacteriologically positive results were communicated with the hospital for better management.

\section{Consent for publication}

Not applicable.

\section{Competing interests}

The authors declare that they have no competing interests.

\section{Availability of data and materials}

The data supporting the conclusion of the study are included in the manuscript

\section{Funding}

Bahir Dar University financially supports to conduct this study. The funder had no role in the overall design and conduction of the study.

\section{Authors' contributions}

HHD: Concept and designed the study, performed the laboratory work, collected and managed the data, analyzed and interpreted the results and contributed to the scientific content of the manuscript. AM: supervised and facilitated the data collection and management, interpreted results, drafted the manuscript and significantly contributed to the scientific content of the study. MK: interpreted results, drafted, critically reviewed, edited and significantly contributed to the scientific content of the manuscript. WM designed and supervised the study, facilitated the data collection and management, analyzed data, interpreted results, drafted the manuscripts and significantly contributed to the scientific content of the study. All authors read and approved the final manuscript.

\section{Acknowledgements}

Our gratitude goes to Bahir Dar University, College of Medicine and Health Sciences, Department of Medical Laboratory Science for the opportunity to undertake this study and the provision of ethical clearance. We also thank the parents/guardians of study participants. The insightful guidance of Mr. Ahmed Hashi (head of Laboratory at Hargeisa Group of Hospital) and the technical support from Mr. Mukhtar Botan and Ms. Ugbad Ibrahim at Hargeisa Group of Hospital are also acknowledged.

\section{References}

1. Abraham ZS, Bazilio J, Kahinga AA, Manyahi J, Ntunaguzi D, Massawe ER. Prevalence and bacteriology of tonsillitis among patients attending otorhinolaryngology Department at Muhimbili National Hospital, Dar es Salaam-Tanzania. Med J Zambia. 2019;46(1):33-40. 
2. Bhalla K, Bhardwaj P, Gupta A, Mehra S, Nehra D, Nanda S. Role of epidemiological risk factors in improving the clinical diagnosis of streptococcal sore throat in pediatric clinical practice. J Family Med Prim Care. 2019;8(10):3130-5.

3. Agrawal A, Kumar D, Goyal A, Gupta R, Bhooshan S. Bacteriological evaluation and their antibiotic sensitivity pattern in tonsillitis. IOSR J Dental Med Sc. 2014;13(3):51. - .55.

4. Ingale MH, Mahajan GD. Evaluation of antibiotic sensitivity pattern in acute tonsillitis. Int J Otorhinolaryngol Head Neck Surg. 2018;4(5):1162.

5. Rebnord IK, Sandvik H, Mjelle AB, Hunskaar S. Factors predicting antibiotic prescription and referral to hospital for children with respiratory symptoms: secondary analysis of a randomised controlled study at out-of-hours services in primary care. BMJ Open. 2017;7(1):e012992.

6. Sadoh WE, Sadoh AE, Oladipo AO, Okunola OO. Bacterial isolates of tonsillitis and pharyngitis in a paediatric casualty setting. $J$ Med Biomed Res. 2008;7:(1-2).

7. Wayne PA. Clinical and Laboratory Standards Institute (CLSI). Performance Standards for Antimicrobial Susceptibility Testing. USA. 2019.

8. Tesfaw G, Kibru G, Mekonnen D, Abdissa A. Prevalence of group A $\beta$-haemolytic Streptococcus among children with pharyngitis in Jimma town, Southwest Ethiopia. Egyptian J ENT Allied Science. 2015;16(1):35-40.

9. Kvestad E, Kværner KJ, Røysamb E, Tambs K, Harris JR, Magnus P. Heritability of recurrent tonsillitis. Arch Otolaryngol Head Neck Surg. 2005;131(5):383-7.

10. Shah R, Bahauddin M, Islam MR, Islam MS, Sadlee SM, Kamal M. Chronic Tonsillitis and its Relation with Childhood Asthma. Int J Sci Res. 2014;245:55.

11. Thors V, Morales-Aza B, Pidwill G, Vipond I, Muir P, Finn A. Population density profiles of nasopharyngeal carriage of 5 bacterial species in pre-school children measured using quantitative PCR offer potential insights into the dynamics of transmission. Hum Vaccin Immunother. 2016;12(2):375-82.

12. Pereira LM, Juman S, Bekele I, Seepersadsingh N, Adesiyun AA. Selected bacterial recovery in Trinidadian children with chronic tonsillar disease. Revista Brasileira de Otorrinolaringologia. 2008;74(6):903-11.

13. Al Ahmary MS, Al Mastour AS, Ghnnam WM. The microbiology of tonsils in Khamis civil hospital, Saudi Arabia. International Scholarly Research Notices. 2012; 2012.

14. Mulu W, Yizengaw E, Alemu M, Mekonnen D, Hailu D, Ketemaw K, et al. Pharyngeal colonization and drug resistance profiles of Morraxella catarrrhalis, Streptococcus pneumoniae, Staphylococcus aureus, and Haemophilus influenzae among HIV infected children attending ART Clinic of Felegehiwot Referral Hospital, Ethiopia. PloS One. 2018;13(5):e0196722.

15. Adegbiji WA. Bacteriology of Tonsillitis among Children Attending Ear, Nose and Throat Department of Ekiti State University Teaching Hospital, Nigeria. J Otolaryngol. 2020;3(1):132.

16. Fox JW, Marcon MJ, Bonsu BK. Diagnosis of streptococcal pharyngitis by detection of Streptococcus pyogenes in posterior pharyngeal versus oral cavity specimens. J Clin Microbiol. 2006;44(7):2593-4.

17. Almadori G, Bastianini L, Bistoni F, Paludetti G, Rosignoli M. Microbial flora of surface versus core tonsillar cultures in recurrent tonsillitis in children. Int J Pediatr Otorhinolaryngol. 1988;15(2):157-62.

18. Bassili A, Barakat S, Sawaf GE, Zaher S, Zaki A, Saleha EE. Identification of Clinical Criteria for Group A- $\beta$ Hemolytic Streptococcal Pharyngitis in Children Living in a Rheumatic Fever Endemic Area. J Trop Pediatr. 2002;48(5):285-93.

19. Jasir A, Noorani A, Mirsalehian A, Schalen C. Isolation rates of Streptococcus pyogenes in patients with acute pharyngotonsillitis and among healthy school children in Iran. Epidemiol Infect. 2000;124(1):47-51.

20. Bazzaz AA, Chelebi NA. Bacteriological evaluation and antibiotic sensitivity pattern in tonsillitis among university students in Kerkuk province, Iraq. J Allergy Clin Immunol Pract. 2018; 1-28.

21. Kalaiarasi R, Subramanian KS, Vijayakumar C, Venkataramanan R. Microbiological profile of chronic tonsillitis in the pediatric age group. Cureus. 2018; 10 (9).

22. Telmesani AM, Ghazi HO. A study of group a streptococcal bacteria isolation from children less than 12 years with acute tonsillitis, pharyngitis and healthy primary school children. J Fam Commun Med. 2002;9(2):23.

23. Anja A, Beyene G, Daka D. Asymptomatic pharyngeal carriage rate of Streptococcus pyogenes, its associated factors and antibiotic susceptibility pattern among school children in Hawassa town, southern Ethiopia. BMC Res Notes. 2019;12(1):1-6.

Page $19 / 22$ 
24. Cavalcanti VP, Camargo LA, Moura FS, Melo Fernandes EJ, Lamaro-Cardoso J, Braga CA, et al. Staphylococcus aureus in tonsils of patients with recurrent tonsillitis: prevalence, susceptibility profile, and genotypic characterization. Braz J Infect Dis. 2019;23(1):8-14.

25. Niedzielski A, Korona-Glowniak I, Malm A. High prevalence of Streptococcus pneumoniae in adenoids and nasopharynx in preschool children with recurrent upper respiratory tract infections in Poland-distribution of serotypes and drug resistance patterns. Medical science monitor: International medical journal of experimental clinical research. 2013;19:54.

26. Malfroot A, Verhaegen J, Dubru JM, Van Kerschaver E, Leyman S. A cross-sectional survey of the prevalence of Streptococcus pneumoniae nasopharyngeal carriage in Belgian infants attending day care centres. Clin Microbiol Infect. 2004;10(9):797-803.

27. Wada FW, Tufa EG, Berheto TM, Solomon FB. Nasopharyngeal carriage of Streptococcus pneumoniae and antimicrobial susceptibility pattern among school children in South Ethiopia: post-vaccination era. BMC Res Notes. 2019;12(1):1-6.

28. Principi N, Marchisio P, Schito GC, Mannelli S. Risk factors for carriage of respiratory pathogens in the nasopharynx of healthy children. Pediatr Infect Dis J. 1999;18(6):517-23.

29. Bhatta DR, Hamal D, Shrestha R, Parajuli R, Baral N, Subramanya SH, et al. Nasal and pharyngeal colonization by bacterial Pathogens: A comparative study between preclinical and clinical sciences medical students. Can J Infect Dis Med Microbiol. 2018; 2018.

30. Garba BI, Muhammad AS, Mohammed F, Onazi SO, Ahmad MM, Yusuf T. Antibiogram of pharyngeal isolates of children with pharyngotonsillitis in a specialist hospital in GUSAU, North-western Nigeria. Kanem Journal of Medical Sciences. 2017;2(11):102-7.

31. Lima AB, Leão LS, Oliveira LS, Pimenta FC. Nasopharyngeal Gram-negative bacilli colonization in Brazilian children attending day-care centers. Braz J Microbiol. 2010;41(1):24-7.

32. Loganathan A, Arumainathan UD, Raman R. Comparative study of bacteriology in recurrent tonsillitis among children and adults. Singapore Med J. 2006;47(4):271.

33. Farida H, Severin JA, Gasem MH, Keuter M, van den Broek P, Hermans PW, et al. Nasopharyngeal carriage of Klebsiella pneumoniae and other Gram-negative bacilli in pneumonia-prone age groups in Semarang, Indonesia. J Clin Microbiol. 2013;51(5):1614-6.

34. Brook I, Gober AE. Increased recovery of Moraxella catarrhalis and Haemophilus influenzae in association with group A $\beta$ haemolytic streptococci in healthy children and those with pharyngo-tonsillitis. J Med Microbiol. 2006;55(8):989-92.

35. Prates M, Tamashiro E, Proenca-Modena JL, Criado MF, Saturno TH, Oliveira AS, et al. The relationship between colonization by Moraxella catarrhalis and tonsillar hypertrophy. Can J Infect Dis Med Microbiol. 2018; 2018.

36. Navne JE, Børresen ML, Slotved HC, Andersson M, Melbye M, Ladefoged K, et al. Nasopharyngeal bacterial carriage in young children in Greenland: a population at high risk of respiratory infections. Epidemiol Infect. 2016;144(15):3226-36.

37. Chochua S, D'Acremont V, Hanke C, Alfa D, Shak J, Kilowoko M, et al. Increased nasopharyngeal density and concurrent carriage of Streptococcus pneumoniae, Haemophilus influenzae, and Moraxella catarrhalis are associated with pneumonia in febrile children. PLoS One. 2016;11(12):e0167725.

38. Lafontaine ER, Wall D, Vanlerberg SL, Donabedian H, Siedjeski DD. Moraxella catarrhalis coaggregates with Streptococcus pyogenes and modulates interactions of $S$. pyogenes with human epithelial cells. infect Immun. 2004; 72:6689-6693.

39. Zautner AE, Krause M, Stropahl G, Holtfreter S, Frickmann H, Maletzki C, et al. Intracellular persisting Staphylococcus aureus is the major pathogen in recurrent tonsillitis. PloS One. 2010;5(3):e9452.

40. Javaeed A, Khan SH, Haqsubhani R, Ghauri SK, Jahan S. Methicillin resistant Staphylococcus aureus prevalence of nasal carriage among healthy MBBS students of Continental Medical College, Lahore. Pakistan J Medical Health Sci. 2016;10(3):1057-9.

41. Hirakata Y, Yanagihara K, Miyazaki Y, Tomono K, Kobayashi I, Kohno S. Antimicrobial susceptibilities of potential bacterial pathogens in adults with acute respiratory tract infections: Prospective Epidemiological Network Investigating Communityacquired Infection SurveiLLance In Nagasaki (PENICILLIN) Study. Diagn Microbiol Infect Dis. 2005;51(4):271-80.

42. Tigabu A, Tiruneh M, Mekonnen F. Nasal Carriage Rate, antimicrobial susceptibility pattern, and associated factors of Staphylococcus aureus with special emphasis on MRSA among urban and rural elementary school children in Gondar, Northwest Ethiopia: A Comparative Cross-Sectional study. Adv Prev Med. 2018; 2018: 9364757. 
43. Brook I, Foote PA. Isolation of methicillin resistant Staphylococcus aureus from the surface and core of tonsils in children. Int $\mathrm{J}$ Pediatr Otorhinolaryngol. 2006;70(12):2099-102.

44. Kateete DP, Asiimwe BB, Mayanja R, Mujuni B, Bwanga F, Najjuka CF, et al. Nasopharyngeal carriage, spa types and antibiotic susceptibility profiles of Staphylococcus aureus from healthy children less than 5 years in Eastern Uganda. BMC Infect Dis. 2019;19(1):1-0.

45. Sayyahfar S, Fahimzad A, Naddaf A, Tavassoli S. Antibiotic susceptibility evaluation of group A streptococcus isolated from children with pharyngitis: a study from Iran. Infect Chemother. 2015;47(4):225.

46. ElSayed N, Ashour M, Amine AE. Vancomycin resistance among Staphylococcus aureus isolates in a rural setting. Egypt Germs. 2018;8(3):134.

47. Acharya A, Gurung R, Khanal B, Ghimire A. Bacteriology and Antibiotic Susceptibility Pattern of Peritonsillar Abscess. J Nepal Med Assoc. 2010;49:(178).

48. Wang LM, Qiao XL, Ai L, Zhai JJ, Wang XX. Isolation of antimicrobial resistant bacteria in upper respiratory tract infections of patients. Biotech. 2016;6(2):1-7.

49. Stacevičienè I, Petraitienè S, Vaičiūnienè D, Alasevičius T, Kirslienè J, Usonis V. Antibiotic resistance of Streptococcus pneumoniae, isolated from nasopharynx of preschool children with acute respiratory tract infection in Lithuania. BMC Infect Dis. 2016;16(1):1-8.

50. Daka D, Loha E, Giday A. Streptococcus pneumonia and antimicrobial resistance, Hawassa referral hospital, South Ethiopia. J Med Lab Diagn. 2011;2(3):27-30.

51. Niedzielski A, Korona-Glowniak I, Malm A. High prevalence of Streptococcus pneumoniae in adenoids and nasopharynx in preschool children with recurrent upper respiratory tract infections in Poland-distribution of serotypes and drug resistance patterns. Medical science monitor. Int Med J Exper Clin Res. 2013;19:54.

52. Hoa NQ, Trung NV, Larsson M, Eriksson B, Phuc HD, Chuc NT, et al. Decreased Streptococcus pneumoniae susceptibility to oral antibiotics among children in rural Vietnam: a community study. BMC Infect Dis. 2010;10(1):1-1.

53. Ba-Saddik IA, Munibari AA, Alhilali AM, Ismail SM, Murshed FM, Coulter JB, et al. Prevalence of Group A beta-haemolytic Streptococcus isolated from children with acute pharyngotonsillitis in Aden, Yemen. Trop Med Int Health. 2014;19(4):431-9.

54. Kargoshaie AA, Najafi M, Akhlaghi M, Khazraie HR, Hekmatdoost A. The correlation between tonsil size and academic performance is not a direct one, but the results of various factors. Acta Otorhinolaryngol Ital. 2009;29(5):255.

55. Gehrke T, Scherzad A, Hagen R, Hackenberg S. Risk factors for children requiring adenotonsillectomy and their impact on postoperative complications: a retrospective analysis of 2000 patients. Anaesthesia. 2019;74(12):1572-9.

56. Nayiga I, Okello E, Lwabi P, Ndeezi G. Prevalence of group a streptococcus pharyngeal carriage and clinical manifestations in school children aged 5-15 yrs in Wakiso District, Uganda. BMC Infect Dis. 2017;17(1):1-8.

57. Steer AC, Jenney AW, Kado J, Good MF, Batzloff M, Magor G, et al. Prospective surveillance of streptococcal sore throat in a tropical country. Pediatr Infect Dis J. 2009;28(6):477-82.

\section{Figures}




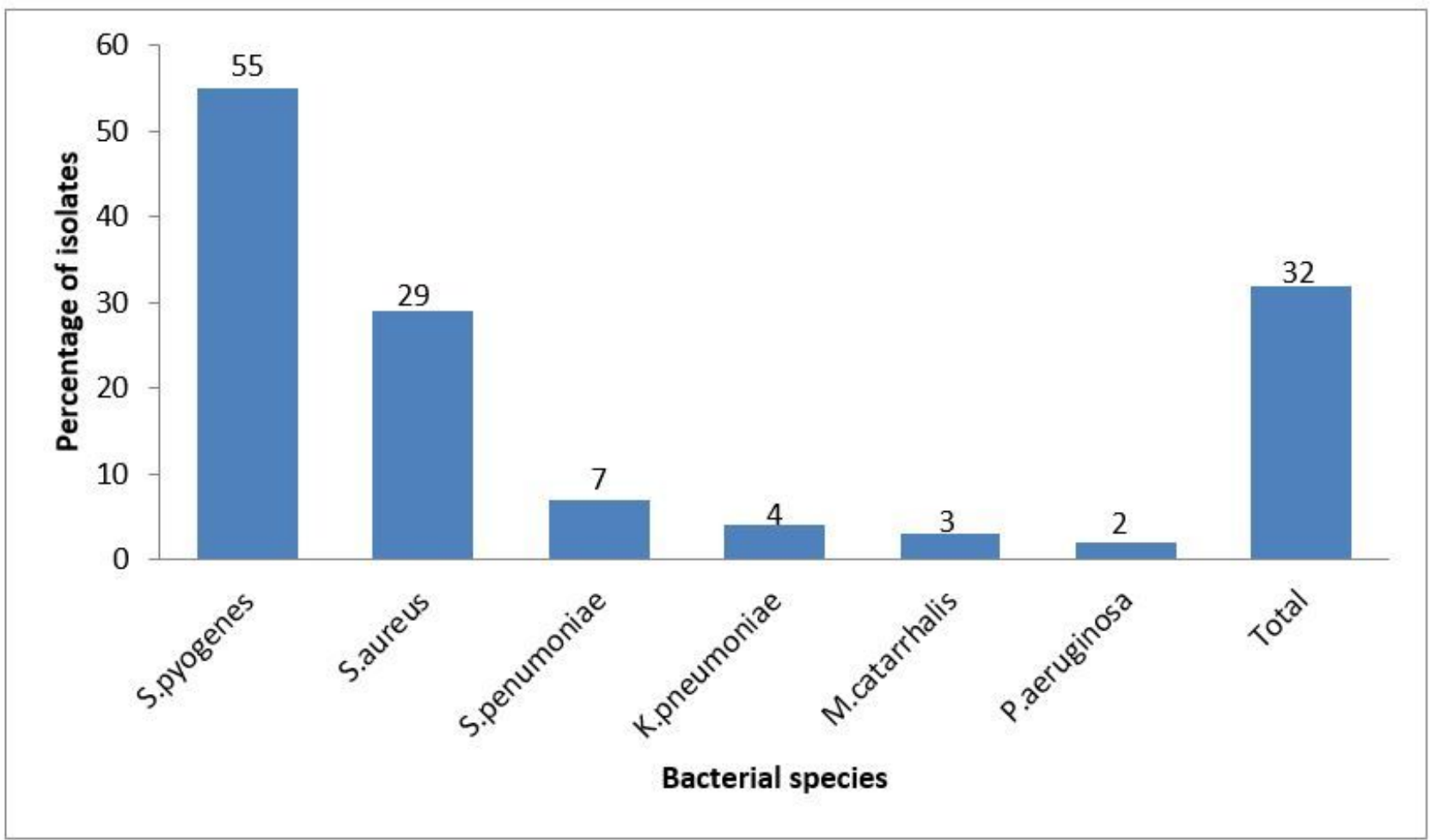

Figure 1

Frequency of bacterial species isolated from Children within five years of age with tonsillitis. 